\title{
Copyright Protection for Digital Images using Singular Value Decomposition and Integer Wavelet Transform
}

\author{
Siddharth Singh \\ Department of Electronics \& Communication University of Allahabad, Allahabad -211002, India \\ E-mail: siddharthjnp@gmail.com \\ Tanveer J. Siddiqui \\ Department of Electronics \& Communication University of Allahabad, Allahabad -211002, India \\ E-mail: tjsiddiqui@allduniv.co.in
}

\begin{abstract}
This paper presents a new technique for copyright protection of images using integer wavelet transform (IWT), singular value decomposition (SVD) and Arnold transform. We divide the cover image into four sub-images by picking alternate pixels from consecutive rows and columns and embed the copyright mark into the sub-image having the largest sum of singular values. The embedding is done by modifying singular values of the IWT coefficients of the selected sub-image. The use of Arnold transform and SVD increases security and robustness against geometric and several signals processing attacks, while IWT provides computational efficiency. We compare the performance of our technique with state-of-the-art-methods. The experimental results show that the proposed technique is more imperceptible and achieves higher security and robustness against various signal processing (filtering, compression, noise addition, histogram equalization and motion blur) and geometrical (cropping, resizing, rotation) attacks.
\end{abstract}

Index Terms - Digital image steganography, copyright protection, singular value decomposition, Arnold transform, integer wavelet transform.

\section{INTRODUCTION}

The rapid development in multimedia technology and computer networks has greatly simplified the sharing, copying and distribution of digital content. At the same time, this poses serious challenges to content authentication and copyright ownership. Digital steganography [1-3] has recently emerged as an attractive technique to cope up these problems. Steganography provides copyright protection by hiding secret information, called copyright mark, in the original content which is later extracted and used to ensure rightful ownership and authentication of the digital content. The copyright mark has to remain invisible and must remain unchanged under geometric operations like rotation, scaling, cropping, and signal processing operations like coding, compression, transformation, noise contamination. Thus, robustness against the geometrical and signal processing operations and imperceptibility are the key characteristics of any steganography technique.

Image steganography technique can be broadly classified into spatial and transform domain. The spatial domain steganography techniques embed the secret data directly into the cover media, whereas the transform domain techniques embed the secret data in the transform coefficients of the cover media. Spatial domain techniques are simple but very sensitive to geometrical as well as signal processing attacks, while the transform domain techniques are computationally expensive but more imperceptible and robust to different attacks.

The copyright protection techniques have already been proposed by a number of authors [4-8][24]. The work involving wavelet transform and singular value decomposition (SVD) includes [9-13]. The scheme proposed in [9] embeds a gray scale watermark in the low frequency sub-band at the finest level by modifying singular values of the sub-band with the help of watermark singular values. The experimental results demonstrate better visual imperceptibility and robustness against various attacks. Bhatnagar and Raman[10] proposed a semi-blind reference watermarking scheme based on discrete wavelet transform (DWT) and SVD for copyright protection and authenticity. They obtained a reference sub-image from directive contrast and wavelet coefficients of high frequency information of an image. The watermark embedding is done by modifying the singular value of the reference image with the singular values of the watermark. The use of reference image for watermark extraction provides increased security and robustness.

Wang and Chen [11] proposed a hybrid DWT-SVD copyright protection scheme based on K-mean clustering and visual cryptography. The proposed scheme extracts image features of the cover image by applying the DWT and SVD and classifies features into two clusters using K-means clustering. In [12], a dual watermarking scheme based on DWT, wavelet packet transform with best tree, 
and SVD is proposed for copyright protection. The authors used two different gray scale images as watermark and embedded them by modifying singular values of two sub-images having largest sum of singular values. In [13], a hybrid non-blind scheme based on SVD and DWT for copyright protection is proposed which involves modification of singular values of each band of the cover image with the singular values of the watermark. In Singh and Siddiqui [24] redundant wavelet transform and SVD-based technique is proposed for copyright protection.

In this paper, we propose a new image steganography technique for copyright protection using the combination of integer wavelet transform (IWT), SVD and Arnold transform. Discrete wavelet transform maps integer gray level values of an image into floating point wavelet coefficients. This means floating-point computation is required during embedding and recovery. IWT maps integer gray level to integer wavelet coefficient and hence operations needed during embedding and recovery involves only integer operation. Thus, the use of the IWT reduces the computational complexity of the proposed scheme. In order to improve security, we scramble the copyright marks using Arnold transform. Scrambling reduces the spatial correlation. Consequently, after embedding the logo information is distributed over the entire spectrum of the cover image. This makes the proposed scheme more robust against geometrical and noise attacks.

Our approach is different from the work reported in [10] and [12] in that unlike their work (i) we use integer wavelet transform (ii) scramble the copyright mark (which is a binary logo) before embedding and (iii) instead of embedding SVD coefficients of logo image, we embed the scrambled logo image itself. This together with IWT makes our scheme computationally competitive. The scrambling introduces randomness and hence security of the logo is preserved.

The proposed technique is tested over six standard cover images (Lena, Pepper, and Lake, Goldhill, Bridge and Pirate images) and three logo images. The performance of the proposed technique is measured in terms of peak signal to noise ratio (PSNR) and correlation coefficient (CC). The experimental results show that the proposed technique achieves higher security and robustness against various signal processing (filtering, compression, noise addition, histogram equalization and motion blur) and geometrical (cropping, resizing, rotation) attacks. The quantitative comparison of the proposed method has been done with Bhatnagar and Raman [10] and Rawat and Raman [12] in terms of PSNR and CC. Instead of using dual watermark, the comparison with [12] has been done using only one of the cover (Goldhill) and logo image (the circle image) reported in [12].

The rest of the paper is organized as follows: we begin by providing necessary basics of IWT, SVD, and Arnold transform in Section 2. The proposed scheme is discussed in Section 3. Experiments and results are discussed in Section 4. Finally, conclusions are made in Section 5.

\section{BACKGROUND CONCEPTS}

The proposed scheme embeds the copyright in integer wavelet transform domain. The cover image is decomposed using IWT and LL band is used for embedding. In order to make the proposed scheme more robust and secure we apply SVD on transformed coefficients and scramble the logo image using Arnold transform before embedding. In the following subsections, we explain the integer wavelet transform, singular value deposition and Arnold transform.

\section{A. Integer Wavelet Transform}

The use of traditional DWT for data hiding results in high computational complexity and rounding error. IWT $[14,23]$ uses integer coefficients, thereby avoids rounding error, and leads to perfect reconstruction. IWT is obtained with simple modification of DWT implemented using lifting scheme proposed by Sweldens [15] which involves three main steps: splitting, predication, and update. Lifting scheme uses simple filtering operations to modify odd and even sample sequences. The modifications are described by the following expressions [16]:

$$
\begin{gathered}
d[n]=d_{0}[n]-\left\lfloor\frac{s_{0}[n+1]+s_{0}[n]}{2}\right\rfloor \\
s[n]=s_{0}[n]+\left\lfloor\frac{d[n-1]+d[n]+2}{4}\right\rfloor
\end{gathered}
$$

where $s[n], d[n]$ are the even and odd samples respectively. After the IWT, the even samples become low frequency coefficients and the odd samples become high frequency coefficients. The mathematical structure of lifting is based on correlation between data, the sample sequence. This type of correlation is useful for data hiding on the wavelet coefficients and improves the perceptual quality of Stego data [17].

\section{B. Arnold Transform}

Arnold transform is chaotic map that maps from integer plane to integer plane $[18,19]$. This transform is given by the following mathematical relation [19]:

$$
\left[\begin{array}{l}
i^{\prime} \\
j^{\prime}
\end{array}\right]=\left[\begin{array}{ll}
1 & 1 \\
1 & 2
\end{array}\right][i, j]^{T} \bmod N
$$

where $[i, j]^{T}$ denotes the $(i, j)^{t h}$ pixel of the image, $T$ denotes the transpose of matrix, and $N$ is the size of image. This transform is iterative and cyclic in nature i.e., the original image repeats itself after certain number of iteration.

When an image is scrambled using this transform its spatial correlation decreases resulting in a wider spectrum. We scramble the logo image using this transform before 
embedding. This results in spread of its frequency information into the entire spectrum of the cover image. Therefore, the logo information is preserved after geometrical attack such as cropping, resizing, and rotation. Moreover, the security of the embedded logo is also increased.

\section{Singular Value Decomposition (SVD)}

An image $X$ can be considered a $M \times N$ matrix with non-negative scalar values. Like other matrices it can be decomposed using SVD [20] into two orthogonal matrices $U$ and $V$ and a diagonal matrix $S$ of singular values of $X$. This decomposition is mathematically described by the following expression [21]:

$$
X=U * S * V^{T}
$$

The two major advantages of using SVD in image steganography are: first, a small variation of singular values of image does not affect the visual perceptual quality and second, it provides robustness against common signal processing attacks [11]. Zhou and Chen [22] showed that singular values of an image are invariant to geometric distortions such as transpose invariance, flip invariance, rotation invariance, scale invariance and translation invariance.

\section{THE PROPOSED SCHEME}

The proposed scheme consists of two stages: embedding and recovery. In the embedding stage, the cover image is first divided into four equal sized subimages by sub sampling, SVD is applied on each subimage, and then sum of their singular values is calculated. The sub-image having the largest sum is selected for embedding. One-level IWT is applied on the selected sub-image. A binary logo image is used as a copyright mark. To increase the security and robustness, the logo image is scrambled by Arnold transform and then embedded by modifying singular value of LL sub-band of the sub-image. The logo is padded to make its size equal to the LL sub-band. Finally, the stego image is obtained by applying inverse integer wavelet transform (IIWT) and inverse sub-sampling.

In the recovery stage, the stego image is first selected as in the embedding stage, IWT is performed on it, and then embedded data is extracted using SVD of stego and cover sub-image. The cover image is not required for recovery; only singular value matrix of its sub-image used for embedding is needed. The details of the embedding and extraction algorithms are as follows:

\section{A. Embedding}

Step 1. The cover image $X$ of size $N \times N$ is divided it into four sub-images of size $N / 2 \times N / 2$ using following expressions:

$$
\begin{aligned}
& X_{1}(i, j)=X(2 i-1,2 j-1) \\
& X_{2}(i, j)=X(2 i-1,2 j) \\
& X_{3}(i, j)=X(2 i, 2 j-1) \\
& X_{4}(i, j)=X(2 i, 2 j)
\end{aligned}
$$

where $i=1,2 \ldots . N / 2$ and $j=1,2 \ldots . N / 2$

Then we decompose these sub-image using SVD and calculate sum of their singular values. The sub-image having maximum singular values, say $Y$, is selected for embedding.

Step 2. One level IWT is performed on $Y$ to decompose it into $Y_{L L}, Y_{L H}, Y_{H L}, Y_{H H}$ and then SVD is applied on its LL sub-band, $Y_{L L}$ :

$$
Y_{L L}=U_{Y L} * S_{Y L L} * V_{Y L}
$$

Step 3. Arnold transform is applied on logo image (L) 20 times to get scrambled logo image $\left(\mathrm{L}_{\mathrm{s}}\right)$ which is embedded into singular matrix $S_{Y L}$ of transform coefficients of LL sub-bands of $Y$ follows:

$$
S_{Y L}^{\prime}=S_{Y L}+k^{*} L_{S}
$$

where $k$ is the gain factor used to specify the strength of embedding data. The value of gain factor is set to 0.02 empirically. Please note that the size of $L_{s}$ is less than $Y_{L L}$. We deal with this by padding extra bits to it.

Step 4. Inverse SVD and inverse integer wavelet transform is applied on $S_{Y_{L L}}^{\prime}$ and then inverse subsampling is done of the resulting image to get stego image $X_{s}$.

\section{B. Recovery}

Step 1. The stego image $X_{S}$ is divided into four subimages using equation (5) and the sub-image, say $Y_{s}$, is selected as in step 1 of embedding stage.

Step 2. One level IWT is performed on $Y_{s}$ and its LL sub-band, $Y_{S_{L L}}$, is decomposed into three orthogonal matrices using SVD as follows:

$$
Y_{s_{L L}}=U_{Y_{s_{L L}}} * S_{Y_{S_{L L}}} * V_{Y_{s_{L L}}}
$$

Step 3. The embedded data, which corresponds to extracted scrambled logo, is extracted using $S_{Y_{S_{L L}}}$ and $S_{Y_{L L}}$

$$
E_{S}=\frac{S_{Y_{S L}}-S_{Y L L}}{k}
$$


where $S_{Y_{S_{L L}}}, S_{Y_{L L}}$ are singular values of stego and cover sub-image respectively.

Step 4. The extra bits appended during embedding is removed and then inverse Arnold transform is applied with same number of iterations used during embedding to get original logo image.

\section{EXPERIMENTAL RESULTS AND DISCUSSIONS}

We have used a number of standard cover images Lena, Pepper, Lake, Goldhill, Bridge and Pirate of size $512 \times$ 512 and three logo images namely JK, JKAU and circle of size $64 \times 64$. For evaluation of the proposed scheme, we have performed visual and quantitative analysis separately. The performance of the proposed scheme has been tested against various signal processing (filtering, compression, noise addition, histogram equalization and motion blur) and geometrical (cropping, resizing, rotation) attacks. We evaluate the proposed scheme in terms of peak signal to noise ratio $(P S N R)$ and correlation coefficient $(C C)$. Table 2 lists the correlation coefficient between original and recovered copyright marks for the proposed scheme under various signal processing attacks. Table 3 lists the CC obtained for these attacks using the cover images used in [10] and [12]

In general, PSNR is used to measure the perceptual quality of the stego image with respect to the original image. The $P S N R$ of a gray scale image of size $M \times N$ is defined as follows:

$$
P S N R=10 \log _{10}\left(\frac{L^{2}}{M S E}\right)
$$

where, $L$ is the maximum possible gray value of the image i.e. $L=255$ for 8 -bit gray scale image and Mean Square Error (MSE) between the original image $X$ and the stego image $X^{\prime}$ is calculated as follows:

$$
M S E=\frac{1}{M N} \sum_{i=1}^{M} \sum_{j=1}^{N}\left(X(i, j)-X^{\prime}(i, j)\right)^{2}
$$

The correlation coefficient $(C C)$ is used to measure the similarity between original logo image $\left(L_{o}\right)$ and the recovered logo image ( $L_{r}$ ) of size $M \times N$. It is defined as follows:

$$
C C=\frac{\sum_{i=1}^{M} \sum_{j=1}^{N}\left(L_{o}(i, j)-\bar{L}_{o}\right)\left(L_{r}(i, j)-\bar{L}_{r}\right)}{\sqrt{\sum_{i=1}^{M} \sum_{j=1}^{N}\left(L_{o}(i, j)-\bar{L}_{o}\right)^{2}} \sqrt{\sum_{i=1}^{M} \sum_{j=1}^{N}\left(L_{r}(i, j)-\bar{L}_{r}\right)^{2}}}
$$

where $\bar{L}_{o}, \bar{L}_{r}$ are mean of original and recovered logo image.

The performance of the proposed scheme against various geometrical and signal processing attacks is listed in Table 2 .

\section{A. Imperceptibility}

We analyze visual results of the proposed scheme using Lena, Pepper, Lake, Goldhill, Bridge and Pirate images as cover and JK, JKAU and circle as logo. Fig. 1 shows Lena, Lake and Pirate images after embedding JK, JKAU and circle image respectively. It also shows recovered logo image. The PSNR values of these results have been shown in Table 1. Table 1 also compares the PSNR values of the proposed method with the methods proposed in [9], [10] and [12].

From the stego images shown in fig. 1, we notice that the embedding does not cause perceptual degradation. The original cover images and the stego images appear visually similar and thus, imperceptibility is maintained which is one of the key requirements of any embedding scheme. In general, any modification in an image resulting in a PSNR value of more than $30 \mathrm{~dB}$ cannot be perceived by human eyes. The PSNR values of the stego image are more than $57 \mathrm{~dB}$ in all the cases. From Table 1, it can be easily seen that the proposed method has higher value of PSNR than Bhatnagar and Raman [10] for Lena, Pepper, Lake cover image. The proposed method performs better than the methods proposed in [12] and [10] on Goldhill cover image and for Lena, Pirate cover image respectively. The dashed (---) values in Table 1 indicates that the results for these cover images are not available. Higher PSNR values indicate that our scheme is more imperceptible.

\section{B. Filtering}

For evaluating the performance of the proposed scheme against filtering attack, we have chosen average and median filter. The used filter size is $13 \times 13$. Fig. 2 shows the Stego Lake and Pirate images after average filter attack and median filter attack. After applying these filters, the quality of stego images degrades but the recovered images have sufficient visual quality as shown in Fig. 2(a)-(d) and preserve most of the geometrical and visual information of the embedded logo. As shown in Table 2 and Table 3, the proposed copyright protection method performs better than the methods in [10] [12] for median and average filtering attack on the tested cover images.

\section{Noise}

Image degradation through noise contamination is very common during the distribution and storage through different media. Further, there are intentional attacks as well by the illegal users to destroy the copyright mark. The proposed scheme is tested against both Gaussian and salt and pepper noise. We contaminated stego Lake and Pirate images shown in Fig. 1(b) and (c) with $75 \%$ Gaussian noise and $50 \%$ salt and pepper noise. The correlation coefficient of the recovered copyright mark with the embedded image is listed in Table 2 and the noisy stego Pirate image and the corresponding extracted copyright mark is shown in Fig. 3. Our scheme exhibits higher correlation coefficient than the method proposed in [10] but lower than Rawat and Raman [12]. 
Table 1. PSNR Values of the Proposed Scheme in Ref. [9, 10] and [12]

\begin{tabular}{|c|c|c|c|c|}
\hline & [9] & [10] & [12] & Proposed Scheme \\
\hline Lena (logo IT of [110]) & 31.12 & 43.65 & --- & 57.50 \\
\hline Pepper (logo IT of [10]) & --- & 44.60 & --- & 57.57 \\
\hline Lake (logo IIT of [10] & --- & 41.60 & --- & 58.14 \\
\hline Goldhill (logo circle) & --- & --- & 40.29 & 58.35 \\
\hline Bridge (logo circle) & --- & 42.44 & --- & 58.40 \\
\hline Pirate (logo circle) & 33.69 & 45.53 & --- & 58.42 \\
\hline
\end{tabular}

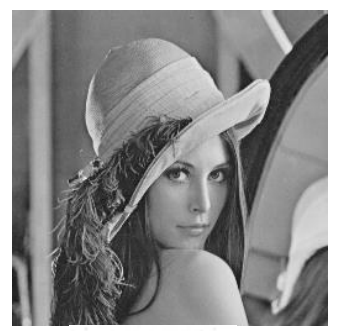

J.K.

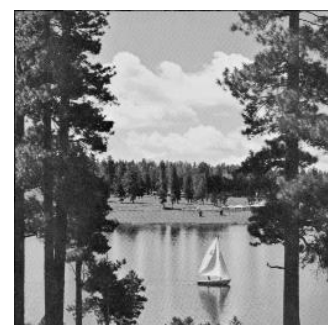

J.K.

A.U

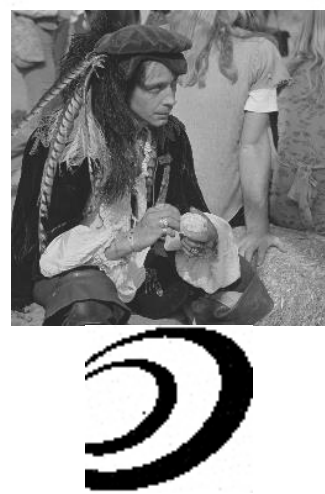

Fig.1. Stego and Recovered Logos Images. a. Lena with logo JK, PSNR $=57.80 \mathrm{~dB}$, b. Lake with logo JKAU, PSNR $=58.14 \mathrm{~dB}$, c. Pirate with logo circle, $\mathrm{PSNR}=58.42 \mathrm{~dB}$
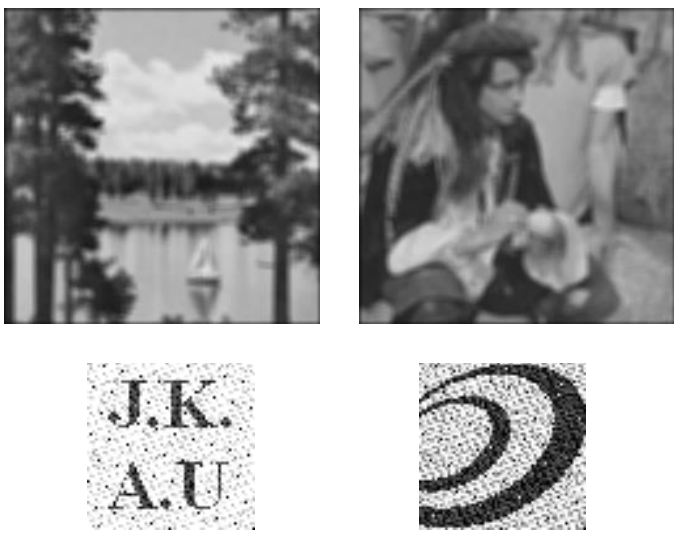
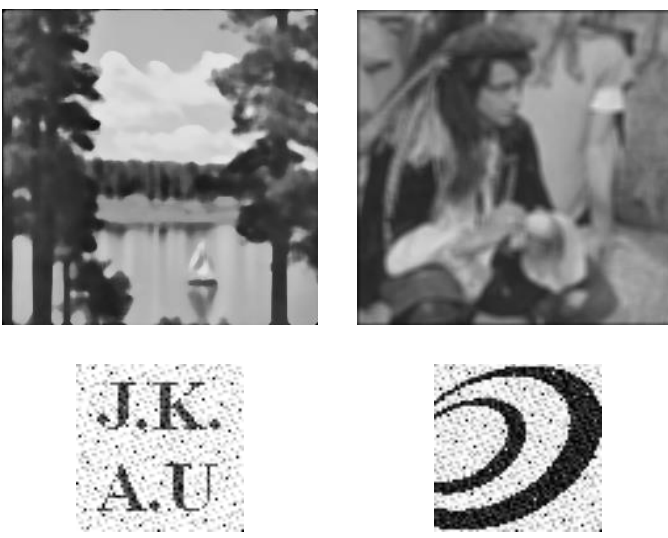

Fig.2. Stego and Recovered Logo Images after Filtering Attacks. a. Stego Lake and Recovered Logo Image After Average Filtering, b. Stego Pirate and Recovered Logo Image After Average Filtering, c. Stego Lake and Recovered Logo Image After Median Filtering, d. Stego Pirate and Recovered Logo Image After Median Filtering
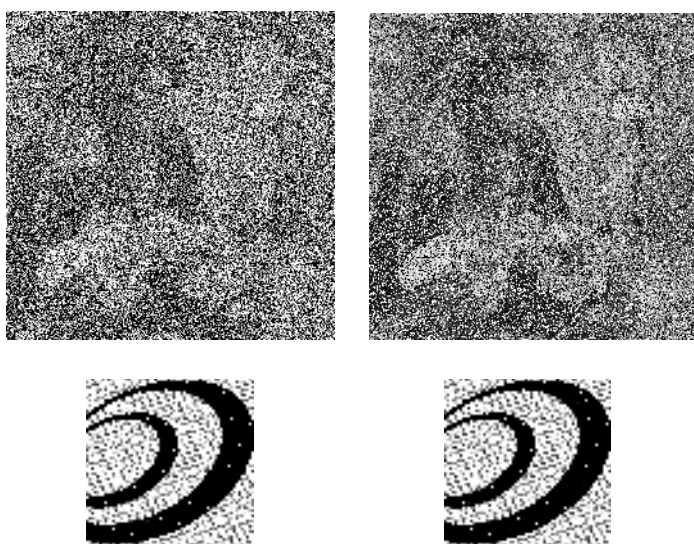

Fig.3. Stego Pirate and Recovered Logo After 75\% Gaussian Noise and $50 \%$ Salt and Pepper Noise 
Table 2. Correlation Coefficient of Extracted Copyright Mark from the Proposed Scheme

\begin{tabular}{ccccccc}
\hline Attack & Lena & Pepper & Lake & Goldhill & Bridge & Pirate \\
Without Attack & 0.9836 & 0.9836 & 0.9779 & 0.9779 & 0.9796 & 0.9796 \\
Avg. Filt. $(13 \times 13)$ & 0.7692 & 0.8408 & 0.8613 & 0.9475 & 0.8592 & 0.8428 \\
Med. Filt. (13×13) & 0.8588 & 0.8731 & 0.9108 & 0.9561 & 0.9461 & 0.9549 \\
Gaussian Noise (75\%) & 0.3464 & 0.3241 & 0.4813 & 0.8558 & 0.8436 & 0.7927 \\
Salt \& Pepper Noise (50\%) & 0.3231 & 0.3020 & 0.4604 & 0.8186 & 0.8046 & 0.7147 \\
Compression (50:1) & 0.8704 & 0.8653 & 0.9138 & 0.9606 & 0.9577 & 0.9598 \\
Compression (80:1) & 0.8719 & 0.8647 & 0.9021 & 0.9577 & 0.9576 & 0.9560 \\
Hist. Equalization & 0.9687 & 0.9611 & 0.9551 & 0.9623 & 0.9554 & 0.9644 \\
Motion Blur & 0.8527 & 0.8588 & 0.8891 & 0.9551 & 0.9365 & 0.9458 \\
Resizing (1/4) & 0.9400 & 0.9330 & 0.9361 & 0.9655 & 0.9598 & 0.9629 \\
Rotation (50 $)$ & 0.9414 & 0.9386 & 0.9307 & 0.9553 & 0.9473 & 0.9529 \\
Cropping (50\%) & 0.3916 & 0.0829 & 0.2509 & 0.9547 & 0.6094 & 0.4093 \\
\hline
\end{tabular}

Table 3. Performance Comparison with Bhatnagar and Raman. [10], and Rawat and Raman[12]

\begin{tabular}{|c|c|c|}
\hline Attack & $\begin{array}{c}\text { Pirate Bhatnagar } \\
\text { and Raman. [10] }\end{array}$ & $\begin{array}{c}\text { Goldhill } \\
\text { Table 1 of } \\
[12])\end{array}$ \\
\hline Without Attack & 0.9996 & 1.0000 \\
\hline Avg. Filt. (13×13) & -0.6209 & 0.3696 \\
\hline Med. Filt. (13×13) & -0.5636 & 0.7057 \\
\hline Gaussian Noise (75\%) & 0.5604 & 0.9376 \\
\hline $\begin{array}{c}\text { Salt \& Pepper Noise } \\
\text { (50\%) }\end{array}$ & --- & 0.9400 \\
\hline Compression (50:1) & --- & 0.9710 \\
\hline Compression (80:1) & 0.9829 & 0.9696 \\
\hline Hist. Equalization & 0.8464 & 0.9880 \\
\hline Motion Blur & 0.5601 & 0.3723 \\
\hline Resizing & 0.0326 & 0.9773 \\
\hline Rotation(50o) & 0.6297 & 0.8692 \\
\hline Cropping (50\%) & -0.2492 & 0.9001 \\
\hline
\end{tabular}

\section{Compression}

Due to the advancement of multimedia technology, a huge amount of digital data is generated. Storage and distribution of this data needs lots of memory and communication bandwidth. For efficient utilization of storage space and bandwidth, data compression is necessary. Compression is one of the most common image processing operations, and hence efficient embedding and recovery method is required to protect the copyright mark against compression.

For performance evaluation of the proposed scheme under compression attack, we used JPEG2000 compression method. We compressed the stego images of Fig. 1(b) and (c) with compression ratio of 50:1 and 80:1. The compressed stego images and the corresponding recovered image are shown in Fig. 4 and the correlation values are given in Table 2. The correlation coefficient values are less but comparable to the methods in [10] and [12] (Table 3).

\section{E. Histogram Equalization and Motion Blur}

We tested the performance of the proposed scheme against the modification introduced in stego image from histogram equalization and motion blur. The histogram equalization of stego images and extracted copyright mark from histogram equalized stego image for Lake image is shown in Fig. 5. The visual information of the extracted copyright mark is better than Bhatnagar and Raman [10]. This can be inferred from the correlation coefficients also. The correlation value of our scheme is better than the method proposed in [10], while slightly less than the method in [12].

We introduce motion blur corresponding to motion angle $45^{\circ}$ and size $20 \times 20$. The motion blurred images and extracted copyright mark for lake image is shown in Fig. 5. The visual appearance and correlation value given in Table 2 of extracted copyright mark from the proposed scheme is better than [10]. The correlation value obtained for blurring attack is better than [12]. 


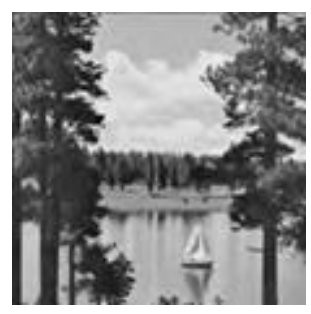

\section{J.K.}
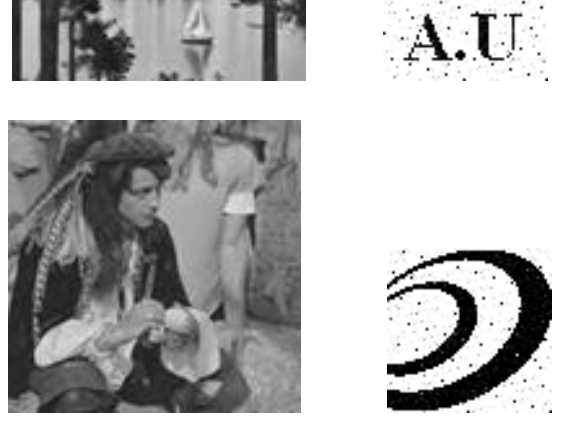

Fig.4. Stego Lake and Pirate Image and Recovered Logo Image after Compression (50:1)

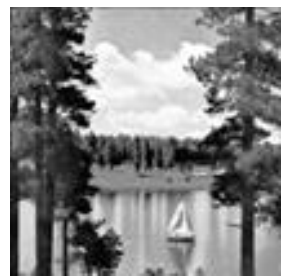

J.K. A.U

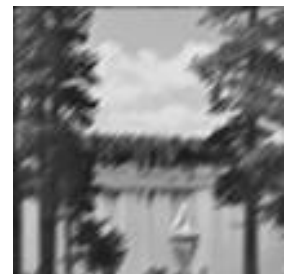

J.K.

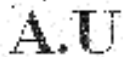

Fig.5. Stego Lake and Recovered Logo after Histogram Equalization and Motion Blur
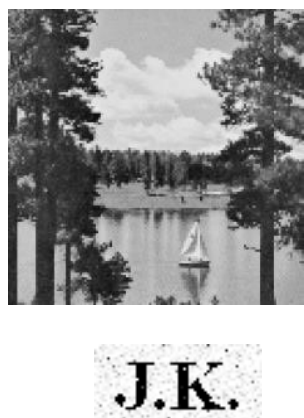

A.U

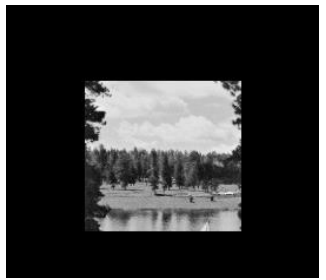

Fig.6. Stego Lake image and recovered logo images after resizing, rotation (50o) and Cropping (50\%)

\section{F. Geometric Attacks}

In this work, we consider the effect of the geometrical operation - resizing, rotation and cropping on stego images. We rescale the stego Lake and Pirate images by $1 / 4$ in both dimension, rotate them by $50 \mathrm{o}$, and crop them by $50 \%$. The results of these geometrical operations with corresponding extracted copyright marks are shown in Figs. 6 respectively. The visual information (shape, edge) of extracted logo is better than Bhatnagar and Raman[10]. Moreover, the proposed scheme is also found better than Bhatnagar and Raman [10] and Rawat and Raman [12] for geometric attacks as shown in Table 2 and table 3. This is because the randomness introduced in copyright mark before embedding decreases the spatial correlation and spreads the copyright mark information in the entire spectrum. Consequently, the information of the copyright mark is distributed to the entire cover image. Hence, the effects of the geometric attacks are reduced.

\section{CONCLUSIONS}

In this paper, we proposed a new secure and robust technique for copyright protection of images using combination of integer wavelet transform (IWT), singular value decomposition (SVD) and Arnold transform. This combination provides higher security with and robustness for copyright protection. The experimental results demonstrate that the proposed scheme is more imperceptible than Bhatnagar et al. [9], Bhatnagar and Raman[10] and Rawat and Raman [12]. The proposed method has been found superior to [the methods presented in 10] and [12] against various (filtering, compression, noise addition, histogram equalization and motion blur) and geometrical (cropping, resizing, rotation) attacks.

\section{REFERENCES}

[1] N. F. Johnson and S. Jajodia, "Exploring Steganography: Seeing the unseen," IEEE Computer, 1998, 31(2), pp. 2634.

[2] Fridrich J., Steganography in Digital Media Principles, Algorithms and Application, Cambridge University Press, New York, 2010.

[3] Cheddad, J. Condell, K. Curran and Mc P. Kevitt, "Digital image Steganography: Survey and analysis of current methods," Review Article, Signal Process., 2010; 90:727752.

[4] C. Chang and J. C. Chung, "An image intellectual property protection scheme for gray-level images using visual secret sharing strategy," Pattern Recognit. Letters, 2002; 23:pp. 931-941.

[5] S. L Hsieh. And B. Y. Huang, "A copyright protection scheme for gray-level images based on image secret sharing and wavelet transformation," Proceedings of International Computer Symposium, 2004, pp. 661-666.

[6] C. S. Hsu and Y. C. Hou, "Copyright protection scheme for digital images using visual cryptography and sampling methods," Optical Engg., 2005.

[7] Wang S.H., Lin Y.P., Wavelet tree quantization for copyright protection watermarking, IEEE Trans. Image Processing, 2004; 13(2): 154-165. 
[8] R. Liu and T. Tan, "An SVD-based watermarking scheme for protecting rightful ownership," IEEE Trans. Multimedia, 2002; 4(1), pp.121-128.

[9] G. Bhatnagar, Q. J. Wu and B. Raman, "A new aspect in robust digital watermarking," Multimedia tools and applications, 2013, vol. 66(2), pp.179-200.

[10] G. Bhatnagar and B. Raman, "A new robust reference watermarking scheme based on DWT-SVD," Comput. Stand. Interfaces, 2009, vol. 31, pp. 1002-1013.

[11] M. S.Wang and W. C. Chen, "A hybrid DWT-SVD copyright scheme based on K-mean clustering and visual cryptography," Comput. Stand. Interfaces, 2009, vol. 31, pp. $750-762$.

[12] S. Rawat and B. Raman, "Best tree wavelet packet transform based copyright protection scheme for digital image," Optics Communi., 2012, vol. 285, pp. 2563-2574.

[13] E. Ganic, A. M. Eskicioglu, "Robust embedding of visual watermarks using DWT-SVD," J. Electronic Imaging, 2005, vol. 14(4), pp. 043004-13.

[14] R Calderbank, I. Daubechies, W. Sweldens and B. L. Yeo, "Wavelet transforms that map integers to integers," Appl. Computation Harmonic Analysis, 1998, 5(3), pp.332-369.

[15] W. Sweldens, "The lifting scheme: a construction of second generation wavelets," SIAM J. Mathematical Analysis. 1998, vol. 29, pp.511-546.

[16] M. D. Adams and F. Kossentini, Reversible integer-tointeger wavelet transforms for image compression: performance evaluation and analysis, IEEE Trans. Image Process., 2000; vol. 9, 1010-1024.

[17] A. Phadikar and S. P. Maity, Data hiding based quality access control of digitals using adaptive QIM and lifting, Signal Process.: Image Communi., 2011; vol. 26, pp. 646661.

[18] V. I. Arnold and A. Avez, "Ergodic problems in classical mechanics, Benjamin," New York, 1968.

[19] R. Ye, "A novel chaos-based image encryption scheme with an efficient permutation-diffusion mechanism," Optics Communicat., 2011, 284: 5290-5298.

[20] R. Kakarala and P. O. Ogunbona, "Signal analysis using a multiresolution form of the singular value decomposition," IEEE Trans. Image Processing, 2001, vol. 10, pp. 724735

How to cite this paper: Siddharth Singh, Tanveer J. Siddiqui,"Copyright Protection for Digital Images using Singular Value Decomposition and Integer Wavelet Transform", International Journal of Computer Network and Information Security(IJCNIS), Vol.8, No.4, pp.14-21, 2016.DOI: 10.5815/ijcnis.2016.04.02
[21] C. C. Chang, P. Tsai and C. C.Lin, "SVD-based digital image watermarking scheme," Pattern Recognit. Letters, 2005, vol.26, pp. 1577-1586.

[22] B. Zho and J. Chen. "A Geometric Distortion Resilient image Watermarking Algorithm Based SVD," Chinese J. Image Graphics, 2004, vol. 9, pp.506-512.

[23] M. Arsalan, S. A. Malik and A. Khan, Intelligent reversible watermarking in integer wavelet domain for medical images, J. of System and Software, 2012, vol. 85(4), pp. 883-894.

[24] S. Singh and T. J. Siddiqui, Robust Image Data Hiding Technique for Copyright Protection, International Journal of Information Security and Privacy, 2013, vol.7(2), pp. 44-56.

\section{Authors' Profiles}

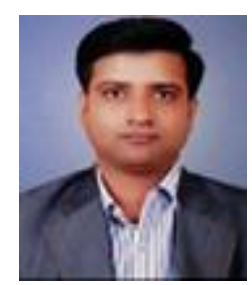

Dr. Siddharth Singh received his Ph.D degree from University of Allahabad, Allahabad (India) in 2015. He is currently Assistant Professor with the Department of Electronics and Communication at NIT Delhi. His current research interests cover Steganography, Digital Image and Signal Processing. He has published a number of quality papers in International Journals and Conferences and authored one book chapter.

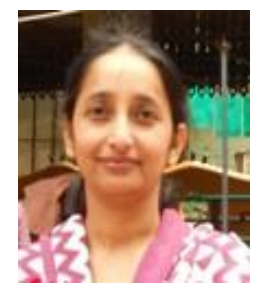

Tanveer J. Siddiqui did her Ph.D. from Allahabad University in the area of Information Retrieval from Allahabad University, India. She is a faculty member at Department of Electronics \& Communication, University of Allahabad. Her research area includes Information Processing and Retrieval. She has got about 40 publications in International Journals and Conferences and authored/edited 5 books \&/conference proceedings. 